\title{
Spatial Relationships over Sparse Representations
}

\author{
Nicolas Loménie*,** \\ University Paris Descartes**, \\ LIPADE, Paris, France \\ Email: lomenie at sip-crip5.org
}

\author{
Daniel Racoceanu* \\ CNRS-National University of Singapore*, \\ IPAL laboratory, Singapore
}

\begin{abstract}
New imaging devices provide image data at very high spatial resolution acquisition and throughput rate. In satellite or medical two-dimensional images, high-content and large image issues plead for more high semantic level interactions between the computer vision systems and the end-users in order to leverage the cognitive symbiosis between both systems for practical tasks such as clinical disease grading practices based on visual inspection. Within the mathematical morphology framework, this seminal paper proposes new theoretical tools to perform high-level spatial relation queries for the exploration of large amount of image data through sparse representations like Delaunay triangulations.
\end{abstract}

\section{INTRODUCTION}

As from now, the images deemed important to be analyzed in the field of computer vision get dramatically in size and content. The notions of high-content or high-throughput are mainstream realities in today image-based sciences. As a matter of fact, end-users of computer vision systems such as biologists or clinicians are more and more in demand of interactive time applications with high-level semantic interactions. These systems are aimed to alleviate the burden of processing such huge amount of data delivered at a high-throughput rate and very high resolution both for satellite images or medical images for instances.

For the sake of illustration, in typical histopathological applications we are currently working on, Tab. I tries to give an idea of the amount of data to be processed by either the clinician or the numerical system. Then, Fig. 1 illustrates the level of details depending of the acquisition resolution. Let us note that images at each of the listed resolution must be stored for clinical and virtual microscopy purposes and that the sample in Fig. 1(b) is a $1024 \times 1280$ pixels sample image at a resolution magnification of $\mathrm{x} 40$ out of over two thousands such samples tiling the Whole Slide Image of Fig. 2(a).

TABLE I

HISTOPATHOLOGICAL IMAGE DATA

\begin{tabular}{|c|c|c|}
\hline Magnification & Size (pixels) & Size (bytes) \\
\hline $\mathrm{x} 1$ & $1018 \times 768$ & $3.05 \mathrm{MB}$ \\
\hline $\mathrm{x} 10$ & $3664 \times 2763$ & $39.54 \mathrm{MB}$ \\
\hline $\mathrm{x} 20$ & $14657 \times 11054$ & $632.48 \mathrm{MB}$ \\
\hline $\mathrm{x} 40$ & $58630 \times 44216$ & $7.77 \mathrm{~GB}$ \\
\hline
\end{tabular}

These figures along with clinical requirements endorse the need for new image analysis toolboxes dedicated to sparse representations of images. Due to large image database issues in the field of object recognition (Ferrari et al. (2006, 2008);

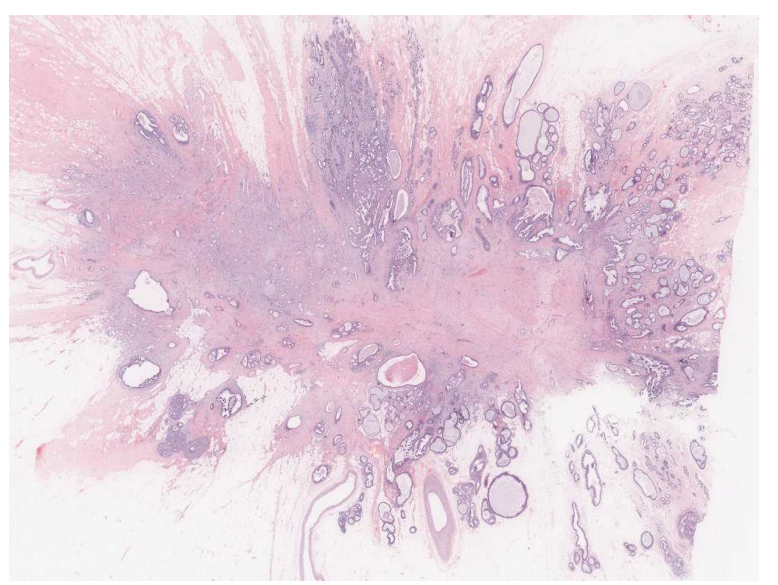

(a)

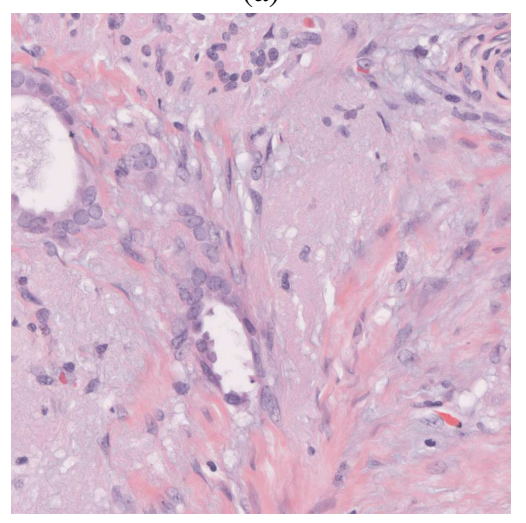

(b)

Fig. 1. (a) Whole Slide Image at resolution $x 1$ (b) Sample image at resolution $\mathrm{x} 40$ out of over 2000 tiling the histopathological image

Shotton et al. (2007)), the representation of image data based on geometrical points-of-interest have already gained momentum in the past years. This is the reason why we consider the set of extracted nuclei as a useful, simplified representation of a microscopic slide (see Fig. 2(a)) on which the system can perform efficient spatial queries. In addition, this simplified architectural/structural representation is required to handle such an amount of pixel data. Working on a restricted point set representation instead of the radiometric Whole Slide Image downsizes this amount of data from around 2.5 billions radiometric color pixels up to about 100000 geometrical 2D points. In the field of classical image analysis, only a few attempts are going in that direction. In Ta et al. (2009), the same kind of 
considerations gave rise to a successful attempt to adapt image processing frameworks -such as Partial Differential Equations based regularization methods- usually acting on radiometric images over a regular grid to unorganized point sets defined within graph representation. From now on, the set of nuclei centroids can be seen as a set of geometrical unorganized points $S \in \Re^{2}$, that is a minimal structural representation of the architecture of the cell distribution in the tissue. Besides, there exist relatively robust ways to detect the nuclei in microscopic images with state-of-the art computer vision algorithms and specific biological markers Cloppet et al. (2008). We recently developed new mathematical morphological operators acting on unorganized point sets that we briefly describe in Section II. Based on this new framework, we propose a new way to perform spatial relation queries on such sparse representations as explained in Section III. This study extends the seminal work of Bloch et al. (2006) on radiometric images to geometric sparse representations of images like Delaunay triangulations.

\section{Morphological Operators on Sparse Data}

In Lomenie \& Stamon (2008), we designed new morphological operators acting on unorganized point set representations and subsequently on mesh representations such as Delaunay triangulations that create the necessary neighborhood system to design mathematical adjunctions like dilations, erosions on such sparse representations. We related these new operators to a computer graphics notion coined $\alpha$-shapes in Edelsbrunner $\&$ Mucke (1994). Hereby we recall the basic elements of the theoretical foundations of this new set of operators and refer the interested reader to the thorough description in Lomenie \& Stamon (2008). From a mathematical point of view, in this seminal paper, we restrict ourself to the set theory framework.

Let $S$ be a point set in $\Re^{2}$. Let $D e l$ be the Delaunay triangulation of the point sites $S$. Then, let us define $\wp(D e l)$ as the set of all the corresponding sub-triangulations $D_{i}$ of $\mathrm{Del}$. As from now, we can define a complete lattice structure for a point set, within the set theory framework, called $\mathcal{L}=(\wp(D e l), \subseteq)$ where $D_{1} \subseteq D_{2}$ denotes the order relation: $\forall T \in D e l, T \in D_{1} \rightarrow T \in D_{2}$.

Let us consider any mesh representation as a set of triangles $T$ with an associated binary value $v l_{\mathrm{T}}$ either 0 or 1 . Then either manually or automatically we can define a sub-mesh of interest associated to an object of interest as exemplified in Fig. 2(b).

To define morphological operators, to each triangle we need to affect values $e_{T}$ and $d_{T}$ in addition to the measure $v a l_{T}$, defined by :

$$
\begin{array}{r}
e_{T}=\min \left\{\operatorname{val}_{T^{\prime}} \mid T^{\prime} \in \nu(T)\right\} \\
d_{T}=\max \left\{\operatorname{val}_{T^{\prime}} \mid T^{\prime} \in \nu(T)\right\}
\end{array}
$$

where $\nu(T)$ is the set of all triangles $T$ of $\mathrm{Del}$ sharing at least one vertex with the triangle $T$, that is :

$$
\nu(T)=\left\{T^{\prime} \in \operatorname{Del} \mid T^{\prime} \cap T \neq \emptyset\right\}
$$

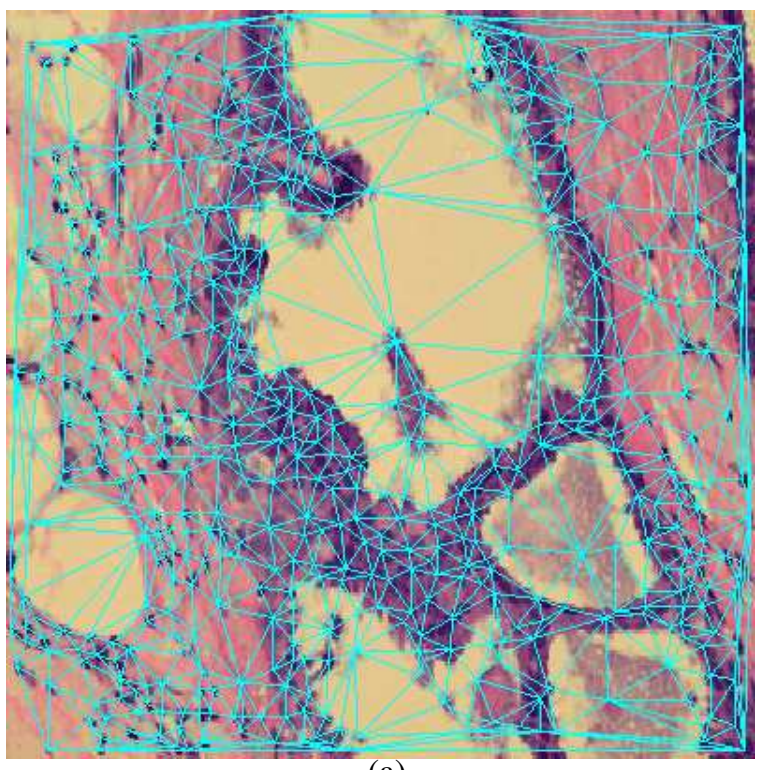

(a)

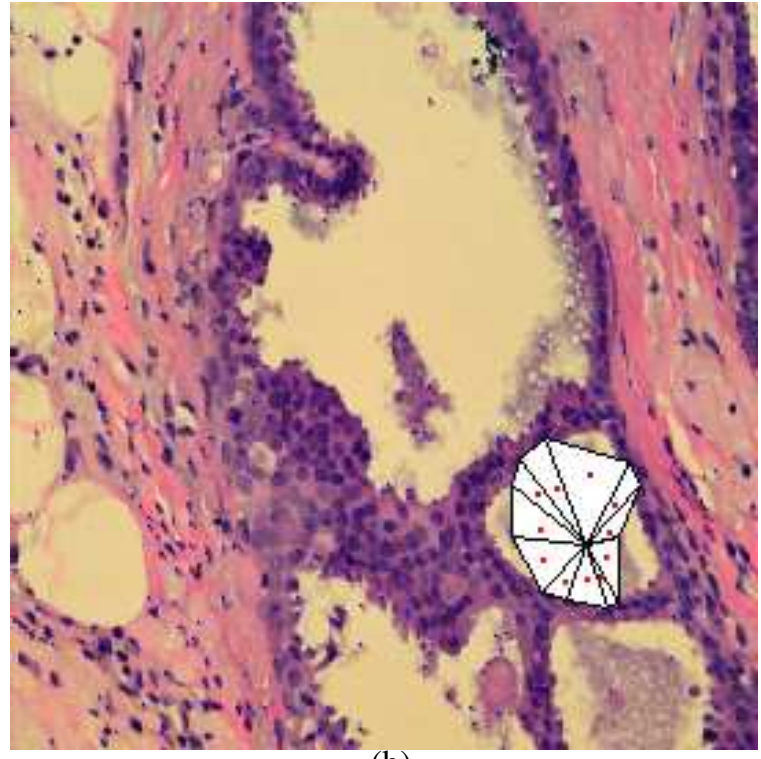

(b)

Fig. 2. A real biological image and (a) the underlying nuclei architecture embedded in a Delaunay triangulation $\operatorname{Del}(S)$ mesh representation and (b) a crisp region of interest as a sub-triangulation of $\operatorname{Del}(S)$. A membership function of 1 is represented by a white triangle and membership values of 0 by transparent triangles

In our case, $\nu(T)$ plays the role of a structuring entity (element or graph - Heijmans et al. (1992)). We proved in Lomenie \& Stamon (2008) that with this definition of a structuring entity the following designed operators $e(D)$ and $d(D)$ are actual mathematical dilation and erosion as defined in the complete lattice framework $\mathcal{L}$ :

$$
\begin{array}{r}
\forall D \in \wp(\text { Del }), \\
e(D)=\left\{T \in \operatorname{Del} \mid e_{T}=1\right\} \\
d(D)=\left\{T \in \operatorname{Del} \mid d_{T}=1\right\}
\end{array}
$$

With these definitions, it is straightforward to extend these 
morphological operators to a functional framework within a real valued lattice structure. Besides, like in classical mathematical morphology theory, we can define these operators within the set theory framework as well. As a matter of fact, for any sub-triangulation $D \in \wp(D e l)$, we can write that $T$ is an interior triangle of $D$ (that is $T \in \operatorname{int}(D)$ ) if there exists a neighborhood of $\mathrm{T}$ which is contained in $D$. Then, with this formal definition $T \in \operatorname{int}(D) \equiv \exists \nu(T) \subset D$ and:

$$
\begin{array}{r}
\forall D \in \wp(D e l), \\
e(D)=\{T \in \operatorname{Del} \mid T \in \operatorname{int}(D)\} \\
d(D)=\left\{T \in \operatorname{Del} \mid T \notin \operatorname{int}(D)^{C}\right\}
\end{array}
$$

From now, having established a sound theoretical framework dedicated to mesh representations, we can derive the whole set of morphological operators including openings, closings and so forth. In particular, we can derive numerical implementations of spatial relation queries over these sparse image representations.

\section{INTERACTIVE SPATIAL QUERY}

In the field of radiometric image analysis, few works have dealt with the effective modeling of spatial relations such as "between", "surround" or "along" Matsakis \& Wendling (1999); Bloch et al. (2006). The major difficulty comes from the high level of contextual references associated to the semantic of these linguistic representations of image content. To us, the more interesting modeling results in the field of image processing can be found in Bloch et al. (2006) for which the use of mathematical morphology operators makes it possible to provide a generic modeling for such spatial reasoning interactions and numerical implementations of such symbolical descriptions.

In this work, our aim is to apply the same kind of considerations to geometrical image representations such as Delaunay triangulations. Usually, these triangulations can be associated with the underlying point-of-interests architecture associated with a radiometric image. We state that these simplified representations are more correlated with the semantic of the images and hence consider that spatial relation reasoning on such representations should be closer to the cognitive spatial reasoning processing performed by the pathologists onto histopathological images.

We developed a Java interface ${ }^{1}$ to test the proposed operators that implements morphological operators acting on Delaunay triangulations described hereby. All these functionalities can be very useful whereby one needs to reason about spatial entities corresponding to unorganized point sets as in the case of extracted nuclei from medical images (see Doyle et al. (2008) for interesting statistical analysis of nuclei architecture embedded in a mesh representation for breast cancer diagnosis).

\footnotetext{
${ }^{1}$ To test the presented results, a Java applet and its source code are available in the public domain at http://sip-crip5.org/lomn/
}

\section{A. The directional dilation}

In order to infer spatial relation reasoning on mesh representations, a useful operator used on regular lattice images is the directional dilation (see Bloch et al. (2006)). We adapt this concept on irregular lattice representations such as Delaunay triangulations. Let $\alpha$ be the directional angle with regard to the horizontal axis of the representation plane.

We define the directional dilation of any mesh $D \in \mathcal{L}$ by modifying the definition of the neighborhood $\nu(T)$ of a triangle $T$ as a directional structural element of direction $\alpha$ that is :

$$
\nu_{\alpha}(T)=\left\{T^{\prime} \in \operatorname{Del} \mid T^{\prime} \cap T \neq \emptyset \text { and } \angle\left(T, T^{\prime}\right)<\alpha+\epsilon\right\}
$$

defining a type of anisotropic neighborhood referred as $\nu_{\alpha}$, to be put in parallel with the isotropic neighborhood definition $\nu_{\text {iso }}$ of equation 2 , where $\alpha$ stand for the angle with the horizontal axis $(O \vec{x})$ and $\epsilon$ is the width or tolerance angle of the structuring element (see Fig. 3). The angle between triangles $T$ and $T^{\prime}$ is given by:

$$
\angle\left(T, T^{\prime}\right)=\angle\left(\overrightarrow{B_{T} B_{T^{\prime}}},(O \vec{x})\right)
$$

where $B_{T}$ is the barycenter of the triangle $T$.

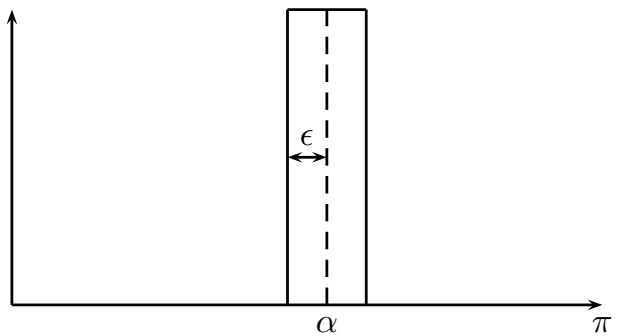

Fig. 3. Crisp angle representation for the directional dilation

Combining Eq. 5 and Eq. 3, we can define the directional dilation as:

$$
\forall D \in \wp(D e l), d_{\alpha, \epsilon}(D)=d_{\nu_{\alpha}}(D)
$$

where $d_{\text {neighborhood }}$ stands for a specific neighborhood system. Whenever $\epsilon$ is not written, $\epsilon=\pi / 8.0$ and $d_{\alpha, \epsilon}=d_{\alpha}$.

\section{B. The relation "left of"}

We can now define a simple absolute directional spatial relation like "left of":

$$
\operatorname{Left}_{d i l}(D)=d_{\pi}^{n}(D) \text { with } n / d_{\pi}^{n}(D)=d_{\pi}^{n+1}(D)
$$

where $d^{n}$ stands for $d \circ d \circ \ldots d n$ times.

Fig. 4(b) illustrates the region "left of". Due to the irregular pavage of the underlying topological space, we advocate that a directional dilation must be filtered out by a final isotropic opening-like operator to get a significant result. As a primary result, any spatial relation defined in this framework must be filtered this way and subsequently we define the "left of" spatial relation as follows:

$$
\operatorname{Left}(D)=d_{\nu_{\text {iso }}} \circ e_{\nu_{i s o}}\left(d_{\pi}^{n}(D)\right) \text { with } n / d_{\pi}^{n}(D)=d_{\pi}^{n+1}(D)
$$


Fig. 4(c) illustrates the final sub-triangulation corresponding to the spatial region "left of" defined over the sparse representation of the image.

\section{The relation "between"}

Based on the proposed mesh-based morphological operators, we consider now the more complex and less objective spatial relation "between" that can be defined as follows:

$$
\begin{array}{r}
\beta_{\text {dil }}\left(D_{1}, D_{2}\right)=d^{n}\left[d^{n}\left(D_{1}\right) \cap d^{n}\left(D_{2}\right)\right] \cap D_{1}^{C} \cap D_{2}^{C} \\
\text { with } \quad n=\inf \left\{k / d^{k}\left(D_{1}\right) \cap d^{k}\left(D_{2}\right) \neq \emptyset\right\}
\end{array}
$$

where $D^{C}$ stands for any involution operator like the set complement operator for this study. And after the necessary post-processing as stated previously, we define the relation "between" over a sparse representation as follows:

$$
\begin{array}{r}
\text { Between }\left(D_{1}, D_{2}\right)=e_{\nu_{i s o}} \circ d_{\nu_{i s o}}\left(\beta_{\text {dil }}\left(D_{1}, D_{2}\right)\right) \\
\text { with } \quad n=\inf \left\{k / d^{k}\left(D_{1}\right) \cap d^{k}\left(D_{2}\right) \neq \emptyset\right\}
\end{array}
$$

Fig. 5 illustrates the achieved results for this specific spatial query over a sparse representation.

\section{CONCLUSION}

The new amount of image data available at very high daily rate and spatial resolution acquisition urge the computer vision community to commit to develop new kind of image analysis tools dedicated to sparse geometrical representations of images and high-level semantic interactions with the novice end-user. We proposed new ways for interacting with huge amount of image data based on a sparse representation like a Delaunay triangulation. Morphological operators previously designed to act on such a sparse and geometrical image representation have been used for efficiently modeling specific spatial relations based on the dilation operator. This work aims at contributing to the definition of a sound theoretical framework dedicated to spatial relation modeling over mesh representations. In addition to the modeling of high-level spatial query useful for the guided exploration or scanning of huge microscopic images, such a numerical modeling of spatial relations can be used to control graph-based image segmentation algorithm based on anatomical or medical knowledge Ta et al. (2009); Colliot et al. (2006). Further work includes the thorough study of an extended spatial relationships set of operators such as "between", "around" as much as the extension to fuzzy versions according to the modeling described in Bloch et al. (2006) for radiometric images.

\section{ACKNOWLEDGMENT}

The authors acknowledge the support of Ludovic Roux IPAL for the cognitive virtual microscope project and Jacques Klossa - TRIBVN for providing histopathological images and inspiring fruitful discussions about the new challenges in microscopic imaging systems.

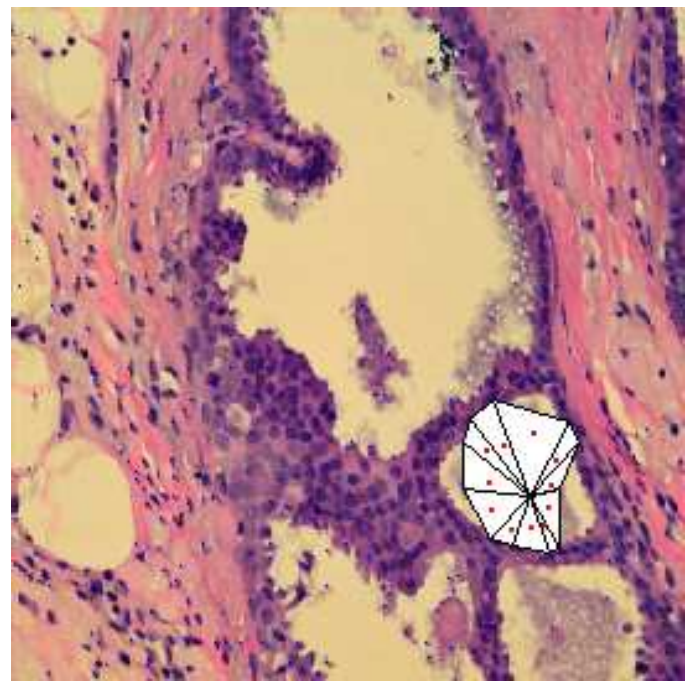

(a)

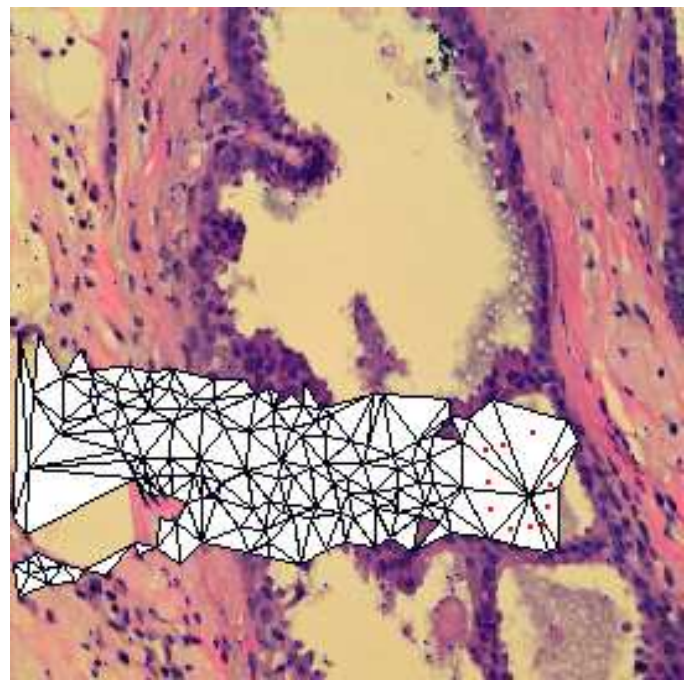

(b)

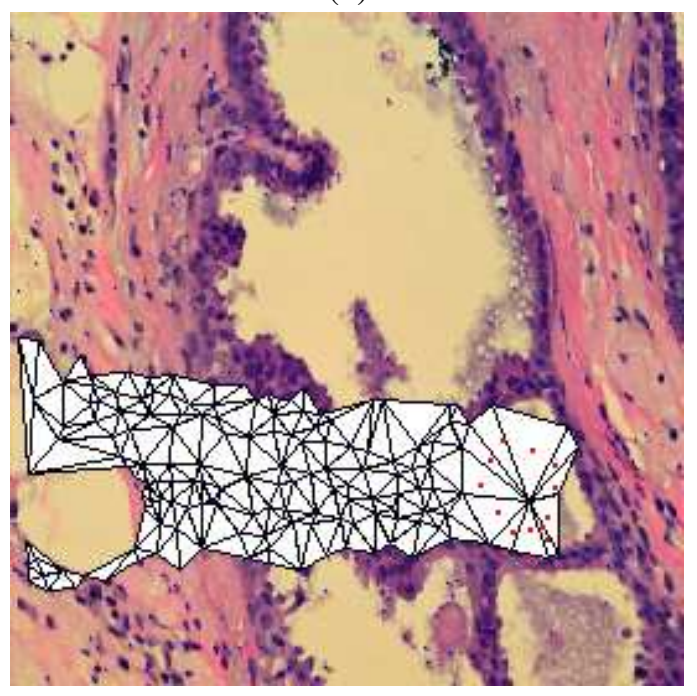

(c)

Fig. 4. (a) One sub-mesh of interest $D$ (b) The left region $\left.\operatorname{Left}_{d i l}(D)\right)$ of the region $D$ (c) The filtered open region left of $D$ with $e_{\nu_{i s o}} \circ$ $d_{\nu i s o}\left(\operatorname{Left}_{d i l}(D)\right)$ 


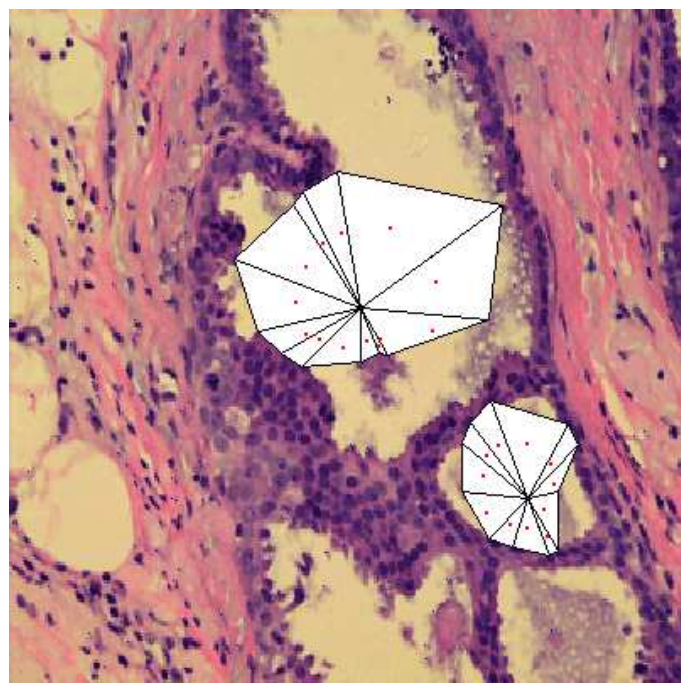

(a)

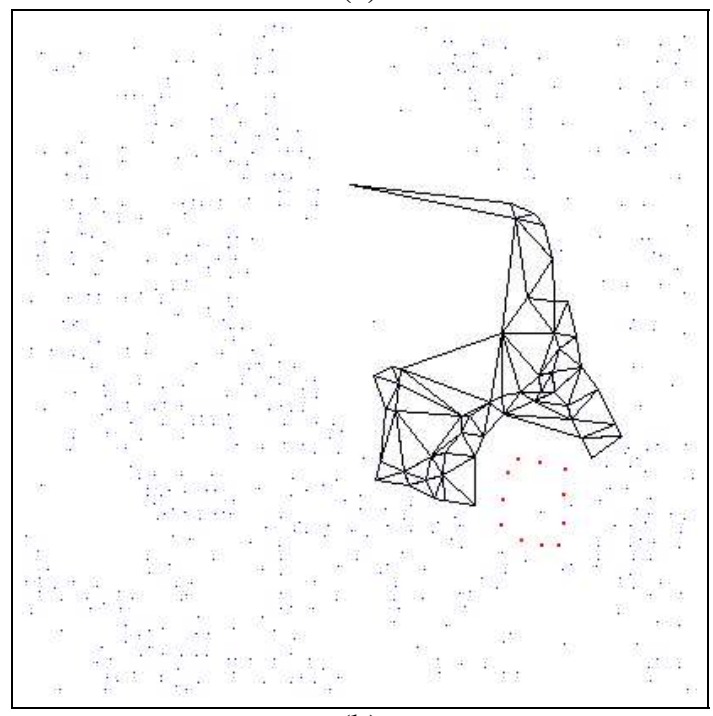

(b)

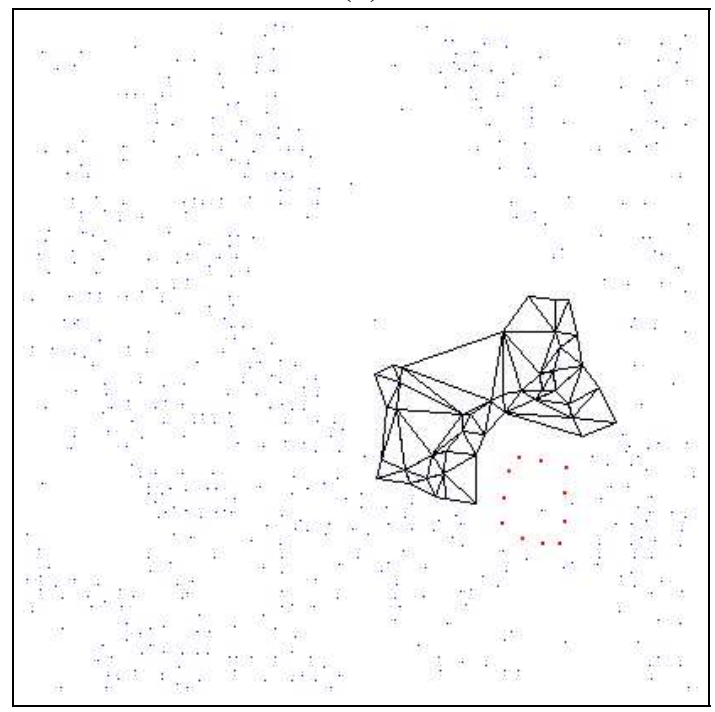

(c)

\section{REFERENCES}

I. Bloch, O. Colliot and R.M Cesar, On the ternary spatial relation "between", IEEE Trans. on Systems, Man, and CyberneticsPart B: Cybernetics, , 36(2), pp. 312-327, 2006.

F. Cloppet and A. Boucher A., Segmentation of overlapping/aggregating nuclei cells in bioimages, 19th International Conference on Pattern Recognition (ICPR08), Tampa, Florida, US, 2008.

O. Colliot, O. Camara and I. Bloch, Integration of Fuzzy Spatial Relations in Deformable Models - Application to Brain MRI Segmentation. Pattern Recognition, 39(8), pp. 1401-1414, 2006.

S. Doyle, S. Agner, A. Madabhushi, M. Feldman and J. Tomaszewski, Automated Grading of Breast Cancer Histopathology Using Spectral Clustering with Textural and Architectural Image Features, in Biomedical Imaging: From Nano to Macro, 2008, ISBI 2008, 5th IEEE International Symposium on, 29, pp. 496-499, 2008.

H. Edelsbrunner and E.P. Mucke, Three-dimensionnal alphashapes, ACM Transactions on Graphics, 13(1), pp. 43-72, 1994.

V. Ferrari, T. Tuytelaars and L. Van Gool, Object Detection by Contour Segment Networks, ECCV, Lecture Notes in Computer Science, 3, pp. 14-28, 2006.

V. Ferrari, L. Fevrier, F. Jurie and C. Schmid, Groups of Adjacent Contour Segments for Object Detection, IEEE Transactions on Pattern Analysis Machine Intelligence, 30(1), pp. 36-51, 2008.

H. Heijmans, P. Nacken, A. Toet and L. Vincent, Graph Morphology, Journal of Visual Communication and Image Representation, 3(1), pp. 24-38, 1992.

N. Lomenie and G. Stamon, Morphological Mesh filtering and alpha-objects, Pattern Recognition Letters, 29(10), pp. 1571-1579, 2008.

P. Matsakis and L. Wendling L., A new way to represent the relative position between real objects IEEE Transactions on Pattern Analysis and Machine Intelligence, 21(7), pp.634643, 1999.

J. Shotton, A. Blake and R. Cipolla, Multi-Scale Categorical Object Recognition Using Contour Fragments IEEE Transactions on Pattern Analysis and Machine Intelligence, 30(7), pp. 1270-1281, 2007.

V.T. Ta, O. Lezoray, A. Elmoataz and S. Schpp Graph-based Tools for Microscopic Cellular Image Segmentation Pattern Recognition, Special Issue on Digital Image Processing and Pattern Recognition Techniques for the Detection of Cancer, 42(6), pp. 1113-1125, 2009.

Fig. 5. (a) Two sub-triangulations of interest $D_{1}$ and $D_{2}$ (b) $\beta_{D i l}\left(D_{1}, D_{2}\right)$ (c) the filtered result after an isotropic opening $\operatorname{Between}\left(D_{1}, D_{2}\right)$ 